\title{
COMMUTANTS FOR SOME CLASSES OF HAUSDORFF MATRICES
}

\author{
B. E. RHOADES
}

(Communicated by Andrew M. Bruckner)

\begin{abstract}
Let $\Gamma$ denote the algebra of all bounded infinite matrices on $c$, the space of convergent sequences, $\Delta$ the subalgebra of $\Gamma$ consisting of lower triangular matrices. It is well known that, if $H$ is any Hausdorff matrix with distinct diagonal entries, then the commutant of $H$ in $\Delta$ contains only Hausdorff matrices. In previous work the author has shown that a necessary condition for the commutant of a Hausdorff matrix $H$ to be the same in $\Gamma$ and $\Delta$ is that $H$ have distinct diagonal entries, but that the condition is not sufficient. In this paper it is shown that certain Hausdorff matrices, with distinct diagonal entries, have the same commutants in $\Gamma$ and $\Delta$.
\end{abstract}

Let $\mathscr{M}$ be the set of all doubly infinite matrices, $\mathscr{B} \subset \mathscr{M}$ the subalgebra of infinite matrices with the finite norm, where $\|B\|:=\sup _{n} \sum_{k}\left|a_{n k}\right|$. Let $\mathscr{T} \subset \mathscr{M}$ be the subalgebra consisting of lower triangular matrices. Hausdorff [7] showed that, if $H$ is any Hausdorff matrix with distinct diagonal entries, then the commutant of $H$ in $\mathscr{T}$ consists of Hausdorff matrices.

Let $\Gamma$ be the subalgebra of $\mathscr{M}$ consisting of all bounded infinite matrices on $c$, the space of convergent sequences, $\Delta$ the subalgebra of $\Gamma$ composed of triangular matrices.

In papers [6] and [7] the author showed that there exist Hausdorff matrices with distinct diagonal entries that have non-Hausdorff matrices in the commutant, and that a necessary condition for the commutant of a Hausdorff matrix in $\Gamma$ and $\Delta$ to be the same is that $H$ have distinct diagonal entries.

The author [6] and Jakimovski [4] have independently shown that the commutant of $(C, 1)$, the Cesàro matrix of order 1 , in $\Gamma$, is $\mathscr{H}$, the set of conservative Hausdorff matrices. (A matrix is conservative if it is convergencepreserving over $c$.) However, the proof only uses the fact that $A,(C, 1) \in \mathscr{B}$. It is well known that, if a Hausdorff matrix has finite norm, then automatically it has all zero column limits, except possibly for the first column, and the first column converges. Since every Hausdorff matrix has row sums equal to $\mu_{0}$, the Silverman-Toeplitz conditions for a matrix to be conservative are satisfied, and the matrix is conservative. Thus the commutants of $(C, 1)$ in $\Delta$ and in $\mathscr{B}$

Received by the editors February 18, 1992 and, in revised form, January 6, 1993, April 14, 1993, and January 26, 1994.

1991 Mathematics Subject Classification. Primary 40G05, 47B47.

Key words and phrases. Cesàro, commutant, Euler, Hausdorff matrices, Hölder.

(C) 1995 American Mathematical Society 
are the same- $\mathscr{H}$. A natural question to ask is, for which Hausdorff matrices, with distinct diagonal entries, is it the case that the commutant in $\mathscr{B}=\mathscr{H}$ ?

The purpose of this paper is to address that question. While we do not answer the question completely, we show that the class of Hausdorff matrices for which the answer is known is fairly large, and includes the Cesàro and Hölder matrices of orders $1,2,3,4$, the known Hausdorff primes, and the Euler matrices. We also provide some examples where the commutants are different.

A Hausdorff matrix $H$ is a lower triangular matrix with entries $h_{n k}=$ $\left(\begin{array}{l}n \\ k\end{array}\right) \Delta^{n-k} \mu_{k}$ for $0 \leq k \leq n$, where $\left\{\mu_{n}\right\}$ is any real or complex sequence and $\Delta$ is the forward difference operator defined by $\Delta \mu_{k}=\mu_{k}-\mu_{k+1}, \Delta^{n} \mu_{k}=\Delta\left(\Delta^{n-1} \mu_{k}\right)$. A necessary and sufficient condition for $H \in \mathscr{B}$ is that $\left\{\mu_{n}\right\}$ have the representation $\mu_{n}=\int_{0}^{1} u^{n} d q(n)$, where $q(u) \in B V(U), U=[0,1]$.

It is the case that every conservative Hausdorff matrix has row sums equal to $\mu_{0}$ and zero column limits, except possibly for the first column.

Suppose $A \in \mathscr{B}$ commutes with $H$. Since $A \in \mathscr{B}$, the rows of $A$ are in $l_{1}$. Then $(A H)_{n k}=(H A)_{n k}$, or

$$
\sum_{j=k}^{\infty} a_{n j} h_{j k}=\sum_{j=0}^{n} h_{n j} a_{j k}, \quad n, k=0,1,2, \ldots
$$

Multiply both sides of $(1)$ by $(1-t)^{k}$, use the representation for $H$, and sum over $k$ to get

$$
\begin{aligned}
& \int_{0}^{1} \sum_{k=0}^{\infty}(1-t)^{k} \sum_{j=k}^{\infty} a_{n j}\left(\begin{array}{l}
j \\
k
\end{array}\right) u^{k}(1-u)^{j-k} d q(u) \\
& =\mu_{n} \sum_{k=0}^{\infty} a_{n k}(1-t)^{k}+\sum_{k=0}^{\infty} \sum_{j=0}^{n-1} h_{n j} a_{j k}(1-t)^{k},
\end{aligned}
$$

since $\sum_{k}\left|a_{j k}\right|<\infty$ for each $j$. Define

$$
g_{n k}(u):=\sum_{j=k}^{\infty} a_{n j}\left(\begin{array}{l}
j \\
k
\end{array}\right) u^{k}(1-u)^{j-k}
$$

Then $g_{n k}(u)$ converges absolutely and uniformly on $U$, and

$$
g_{n k}(u)=\frac{(-1)^{k} u^{k} g_{n 0}^{(k)}(u)}{k !}
$$

Since the rows of $A$ are in $l_{1},\left\{g_{n k}(u)\right\}$ is a uniformly bounded sequence of functions that are continuous on $U$ and analytic for $|u-1|<1$.

For each fixed $u, 0<u \leq 1$,

$$
\begin{aligned}
\sum_{k=0}^{\infty}(1-t)^{k} g_{n k}(u) & =\sum_{k=0}^{\infty} \frac{(-1)^{k} u^{k} g_{n 0}^{(k)}(u)(1-t)^{k}}{k !} \\
& =\sum_{k=0}^{\infty} \frac{g_{n 0}^{(k)}(u)(t u-u)^{k}}{k !} \\
& =g_{n 0}(t u)
\end{aligned}
$$


since, by Taylor's theorem, the series is the expansion of the function $g_{n 0}$, in terms of the variable $t u$, about $t=1$ (i.e., about $u$ ). For $u=0$,

$$
\sum_{k=0}^{\infty}(1-t)^{k} g_{n k}(0)=g_{n 0}(0)=g_{n 0}(t 0) \text {. }
$$

Then (2) takes the form

$$
\int_{0}^{1} g_{n 0}(t u) d q(u)=\mu_{n} g_{n 0}(t)+\sum_{j=0}^{n-1} h_{n j} g_{j 0}(t) .
$$

Suppose that $H$ is such that the solution of (4) for each $n=0,1, \ldots, m-1$ implies that $a_{n k}=0$ for $k>n, 0 \leq n<m$, i.e., from (3), that $g_{n 0}(t)$ is a polynomial of degree $\leq n$. Then, for $n=m$, (4) becomes

$$
\int_{0}^{1} g_{m 0}(t u) d q(u)=\mu_{m} g_{m 0}(t)+P_{m-1}(t),
$$

where $P_{m-1}(t)$ is a polynomial of degree $\leq m-1$.

If one can show that, for $n=0,1,2, \ldots$, and any arbitrary polynomial $P_{n-1}(t)$, every solution of $(5)$ of the form $g_{m 0}(t)=\sum_{k=0}^{\infty} a_{m k}(1-u)^{k}$ with $\left\{a_{m k}\right\}_{k \geq 0} \in l_{1}$ is a polynomial of degree $m$, then $A$ must be triangular and $\operatorname{Com} H$ in $\Gamma=\operatorname{Com} \mathscr{H}$ in $\mathscr{B}=\mathscr{H}$.

Using the remarks about $(5)$, we shall now investigate the commutants in $\mathscr{B}$ for some specific Hausdorff matrices.

The Cesáro matrix of order $\alpha$ has $\mu_{n}=\left(\begin{array}{c}n+\alpha \\ \alpha\end{array}\right)^{-1}$ and is generated by the mass function $q(u)=1-(1-u)^{\alpha}$.

Theorem 1. Let $n$ be a positive integer. Then $\operatorname{Com}(C, n)$ in $\mathscr{B}=\mathscr{H}$ for $n=1,2,3,4$.

Proof. We shall assume $n>1$, since the result is known for $n=1$.

Substituting in (5) and making the change of variable $s=t u$, one obtains

$$
n \int_{0}^{t} g_{m 0}(s)(1-s / t)^{n-1} d s=t\left(\mu_{m} g_{m 0}(t)+P_{m-1}(t)\right) .
$$

Differentiating this expression with respect to $t$, it can be verified that, for each $k<n$,

$$
\begin{gathered}
n(n-1) \cdots(n-k) \int_{0}^{t} g_{m 0}(s)(1-s / t)^{n-k-1} s^{k} d s \\
=\sum_{j=0}^{k} c(k, j) t^{j+k+1}\left(\mu_{m} g_{m 0}^{(j)}(t)+P_{m-1}^{(j)}(t)\right)
\end{gathered}
$$

where the $c(k, j)$ are constants depending in $j$ and $k$.

Setting $k=n-1$ in (6) yields

$$
n ! \int_{0}^{t} g_{m 0}(s) s^{n-1} d s=\sum_{j=0}^{n-1} c(n-1, j) t^{j+n}\left(\mu_{m} g_{m 0}^{(j)}(t)+P_{m-1}^{(j)}(t)\right)
$$


Differentiating again and dividing by $\mu_{m}$ yields

$$
\begin{array}{r}
{\left[\prod_{j=1}^{n}(m+j)\right] g_{m 0}(t) t^{n-1}=\sum_{j=0}^{n-1} c(n-1, j)\left[(j+n) t^{j+n-1}\left(g_{m 0}^{(j)}(t)+\mu_{m}^{-1} P_{m-1}^{(j)}(t)\right)\right.} \\
\left.+t^{j+n}\left(g_{m 0}^{(j+1)}(t)\right)+\mu_{m}^{-1} P_{m-1}^{(j+1)}(t)\right],
\end{array}
$$

or, dividing by $t^{n-1}$ and transposing terms,

$$
\sum_{j=0}^{n} d(n, j) t^{j} g_{m 0}^{(j)}(t)-g_{m 0}(t) \prod_{j=1}^{n}(m+j)=-\mu_{m}^{-1} \sum_{j=0}^{n} d(n, j) t^{j} P_{m-1}^{(j)}(t),
$$

where

$$
d(n, j)=c(n-1, j)(j+n)+c(n-1, j-1),
$$

with the convention that $d(n,-1)=0$.

The homogeneous part of equation (7) is an Euler-type differential equation which is solved by substituting $g_{m 0}(t)=t^{r}$, to obtain a polynomial equation in $r$. Performing this substitution in (7) yields

$$
\sum_{j=0}^{n} c(n, j) t^{j} r(r-1) \cdots(r-j+1) t^{r-j}=t^{r} \prod_{j=1}^{n}(m+j),
$$

or

$$
\sum_{j=0}^{n} c(n, j) r(r-1) \cdots(r-j+1)=\prod_{j=1}^{n}(m+j) .
$$

Lemma 1. $\sum_{j=0}^{n} c(n, j) r(r-1) \cdots(r-j+1)=\prod_{j=1}^{n}(r+j)$ for each $n>1$.

Proof. If one chooses $g(u): t^{r}, q(u)=1-(1-u)^{n}$, then (5) is satisfied with $P_{m-1}(t) \equiv 0$. From (7) we get for this function $t^{r}, \sum_{j=0}^{n} c(n, j) t^{j} r(r-1) \cdots$ $(r-j+1) t^{j-r}-\prod_{j=1}^{n}(r+j)=0$.

We therefore have the indicial equation

$$
\prod_{j=1}^{n}(r+j)=\prod_{j=1}^{n}(m+j)
$$

For the particular nonhomogeneous solution, since the right-hand side of (7) is a polynomial of degree $\leq m-1$, one can use the method of undetermined coefficients to obtain, as solution, a polynomial $Q_{m-1}(t)$ of degree at most $m-1$. We are interested in examining the coefficients of $g_{m 0}(t)$ for $k>m$. Therefore, $Q_{m-1}(t)$ plays no role in the analysis for $n=2,3$, or 4 .

Since $g_{m 0}(t)$ is continuous at $t=0$, we must have $c_{1}=0$. Hence $g_{m 0}(t)$ is a polynomial of degree $m$, and $A$ is triangular.

If $n=3,(10)$ becomes $(r+1)(r+2)(r+3)=(m+1)(m+2)(m+3)$, and the roots are $r=m,-3-m / 2 \pm \gamma i$, where $\gamma=\left(3 m^{2}+12 m+18\right)^{1 / 2} / 2$. Then $g_{m 0}(t)=c_{m 0} t^{m}+c_{m 1} t^{-3-m / 2+\gamma i}+c_{m 2} t^{-3-m / 2-\gamma i}$. Since $g_{m 0}(t)$ is continuous at $t=0$, we must have either $c_{m 1}=c_{m 2}=0$, or some linear combination of $c_{m 1}$ and $c_{m 2}$ that removes the discontinuity at $t=0$. We shall now show that the second situation cannot occur. Without loss of generality we may assume that both $c_{m 1}$ and $c_{m 2}$ are different from zero. For, if one of them is zero, 
then it follows that the other must be zero also. We may write $c_{m 1} t^{-3-m / 2+\gamma i}+$ $c_{m 2} t^{-3-m / 2-\gamma i}=c_{m 2} t^{-3-m / 2-\gamma i}\left(c_{m 1} t^{2 \gamma i} / c_{m 2}+1\right)=c_{m 2} f(t) g(t)$, say. $f$ is not continuous at $t=0$, whereas $g(0)=1$. It is clear that there is no choice of $c_{m 1} / c_{m 2}$ that will remove the discontinuity at $t=0$. Therefore, $c_{m 1}=c_{m 2}=$ $0, g_{m 0}(t)$ is a polynomial of degree $m$ and $A$ is triangular.

If $n=4,(10)$ becomes $(r+1)(r+2)(r+3)(r+4)=(m+1)(m+2)(m+3)$ $\times(m+4)$, which has roots $r=m,-m-5,-5 / 2 \pm \gamma i$, where now $\gamma=$ $\left(4 m^{2}+20 m+15\right)^{1 / 2} / 2$. Then $g_{m 0}(t)=c_{m 0} t^{m}+c_{m 1} t^{-m-5}+c_{m 2} t^{-5 / 2+\gamma i}+$ $c_{m 3} t^{-5 / 2-\gamma i}$. Suppose that $c_{m 1} \neq 0$. Then we may write $c_{m 1} t^{-m-5}+c_{m 2} t^{-5 / 2+\gamma i}+$ $c_{m 3} t^{-5 / 2-\gamma i}=c_{m 1} t^{-m-5}\left(1+\left(c_{m 2} / c_{m 1}\right) t^{5 / 2+\gamma i}+\left(c_{m 3} / c_{m 1}\right) t^{5 / 2-\gamma i}\right)=c_{m 1} f(t) g(t)$, say. Now $f$ is discontinuous at $t=0$, and there is no combination of $c_{m 2} / c_{m 1}$ and $c_{m 3} / c_{m 1}$ that will remove the discontinuity at $t=0$. Therefore $c_{m 1}=0$. Using an argument similar to that employed for $n=3$, it follows that $c_{m 2}=c_{m 3}=0$. Therefore, $g_{m 0}(t)$ is a polynomial of degree $m$, and $A$ is triangular.

We shall now show that the commutant of $(C, 5)$ in $\Gamma$ contains nontriangular matrices. From (4), with $q(u)=1-(1-u)^{5}$, we have

$$
\int_{0}^{1} g_{m 0}(t u) 5(1-u)^{4} d u=\sum_{j=0}^{m} h_{m j} g_{j 0}(t) \text {. }
$$

Making the substitution $s=t u$ yields

$$
\int_{0}^{t} g_{m 0}(s) 5(1-s / t)^{4} d s=\sum_{j=0}^{m} h_{m j} g_{j 0}(t) .
$$

Differentiating, we obtain

$$
5 \int_{0}^{t} g_{m 0}(s) 4(1-s / t)^{3}\left(\frac{s}{t^{2}}\right) d s=\sum_{j=0}^{m} h_{m j}\left[g_{j 0}(t)+t g_{j 0}^{\prime}(t)\right],
$$

or

$$
20 \int_{0}^{t} g_{m 0}(s)(1-s / t)^{3} s d s=\sum_{j=0}^{m} h_{m j}\left[t^{2} g_{j 0}(t)+t^{3} g_{j 0}^{\prime}(t)\right]
$$

Differentiating and then multiplying by $t^{2}$ yields

$$
60 \int_{0}^{t} g_{m 0}(s)(1-s / t)^{2} s^{2} d s=\sum_{j=0}^{m} h_{m j}\left[2 t^{3} g_{j 0}(t)+4 t^{4} g_{j 0}^{\prime}(t)+t^{5} g_{j 0}^{\prime \prime}(t)\right] \text {. }
$$

Another differentiation and multiplication by $t^{2}$ gives

$$
120 \int_{0}^{t} g_{m 0}(s)(1-s / t) s^{3} d s=\sum_{j=0}^{m} h_{m j}\left[6 t^{4} g_{j 0}(t)+18 t^{5} g_{j 0}^{\prime}(t)+9 t^{6} g_{j 0}^{\prime \prime}(t)+t^{7} g_{j 0}^{(3)}(t)\right] \text {. }
$$

Differentiation and multiplication by $t^{2}$ gives

$$
\begin{aligned}
120 \int_{k}^{t} g_{m 0}(s) s^{4} d s=\sum_{j=0}^{m} h_{m j}\left[24 t^{5} g_{j 0}(t)+\right. & 96 t^{6} g_{j 0}^{\prime}(t)+72 t^{7} g_{j 0}^{\prime \prime}(t) \\
& \left.+16 t^{8} g_{j 0}^{(3)}(t)+t^{9} g_{j 0}^{(4)}(t)\right]
\end{aligned}
$$


One more differentiation produces

$$
\begin{array}{r}
120 g_{m 0}(t) t^{4}=\sum_{j=0}^{m} h_{m j}\left[120 t^{4} g_{j 0}(t)+600 t^{5} g_{j 0}^{\prime}(t)+600 t^{6} g_{j 0}^{\prime \prime}(t)+200 t^{7} g_{j 0}^{(3)}(t)\right. \\
\left.+25 t^{8} g_{j 0}^{(4)}(t)+t^{9} g_{j 0}^{(5)}(t)\right]
\end{array}
$$

Thus

$$
\begin{aligned}
& \mu_{m}\left(t^{5} g_{m 0}^{(5)}(t)+25 t^{4} g_{m 0}^{(4)}(t)+200 t^{3} g_{m 0}^{(3)}(t)\right.+600 t^{2} g_{m 0}^{\prime \prime}(t) \\
&\left.+600 t g_{m 0}^{\prime}(t)+120 t g_{m 0}(t)\right) \\
&=120 g_{m 0}(t)-\sum_{j=0}^{m-1} h_{m j}\left[120 g_{j 0}(t)+600 t^{1} g_{j 0}^{\prime}(t)+600 t^{2} g_{j 0}^{\prime \prime}(t)+200 t^{3} g_{j 0}^{(3)}(t)\right. \\
&\left.+25 t^{4} g_{j 0}^{(4)}(t)+t^{5} g_{j 0}^{(5)}(t)\right],
\end{aligned}
$$

or

$$
\begin{aligned}
& t^{5} g_{m 0}^{(5)}(t)+25 t^{4} g_{m 0}^{(4)}(t)+ 200 t^{3} g_{m 0}^{(3)}(t)+600 t^{2} g_{m 0}^{\prime \prime}(t) \\
&+ 600 t g_{m 0}^{\prime}(t)+120\left(1-\mu_{m}^{-1}\right) t g_{m 0}(t) \\
&=\mu_{m}^{-1} \sum_{j=0}^{m-1} h_{m j}\left[120 g_{j 0}(t)+600 t^{1} g_{j 0}^{\prime}(t)+600 t^{2} g_{j 0}^{\prime \prime}(t)\right. \\
&\left.+200 t^{7} g_{j 0}^{(3)}(t)+25 t^{8} g_{j 0}^{(4)}(t)+t^{9} g_{j 0}^{(5)}(t)\right] .
\end{aligned}
$$

Let $h_{m 0}(t)$ and $p_{m 0}(t)$ denote the homogeneous and nonhomogeneous solutions of (11), respectively.

For $n=5,(10)$ becomes $(r+1)(r+2)(r+3)(r+4)(r+5)=(m+1)$ $\times(m+2)(m+3)(m+4)(m+5)$. One obvious root is $r=m$.

The remaining polynomial is

$$
\begin{aligned}
r^{4} & +(m+15) r^{3}+\left(m^{2}+15 m+85\right) r^{2}+\left(m^{3}+15 m^{2}+85 m+225\right) m \\
& +m^{4}+15 m^{3}+85 m^{2}+225 m+274
\end{aligned}
$$

Using a theorem of Routh [1, p. 142], one constructs the array

$$
\begin{array}{lll}
r_{0 j} & 1 \quad m^{2}+15 m+85 & m^{4}+15 m^{3}+85 m+225 m+274 \\
r_{1 j} & m+15 \quad m^{3}+15 m^{2}+85 m+225 \\
r_{2 j} & \frac{15\left(m^{2}+15 m+60\right)}{m+15} \quad m^{4}+15 m^{3}+85 m^{2}+225 m+274 \\
r_{3 j} & -\frac{\left(m^{6}+30 m^{5}+310 m^{4}+600 m^{3}-9,851 m^{2}-68,280 m-140,850\right)}{15\left(m^{2}+15 m+85\right)} \\
r_{4 j} & m^{4}+15 m^{3}+85 m^{2}+225 m+274 .
\end{array}
$$

Note that the polynomial in the numerator of $r_{3 j}$ has one real positive root, which lies between 6 and 7. Thus, for $0 \leq m \leq 6$, there are no sign changes in the first column, indicating that all four roots of the polynomial have negative real parts. Using the now familiar argument, it follows that $a_{m m}=0$ for $n>m, m=1,2, \ldots, 6$. For $m \geq 7$, there are two sign changes, indicating that two of the roots have positive real part, and the remaining two have negative 
real part. Let $\alpha_{m} \pm i \gamma_{m}$ denote the complex roots with positive real part, and $\beta_{m} \pm i \delta_{m}$ denote the complex roots with negative real part. Then $h_{m 0}(t)=$ $c_{m 0} t^{m}+c_{m 1} t^{\alpha_{m}+i \gamma_{m}}+c_{m 2} t^{\alpha_{m}-i \gamma_{m}}+c_{m 3} t^{\beta_{m}+i \delta_{m}}+c_{m 4} t^{\beta_{m}+i \delta_{m}}$. Using the argument for $n=3$, it follows that $c_{m 3}=c_{m 4}=0$. Suppose that $c_{m 2} \neq 0$. Then we may write $c_{m 1} t^{\alpha_{m}+i \gamma_{m}}+c_{m 2} t^{\alpha_{m}-i \gamma_{m}}=c_{m 2} t^{\alpha_{m}-i \gamma_{m}}\left(1-\left(c_{m 1} / c_{m 2}\right) t^{i \gamma_{m}}=c_{m 2} g(t)\right.$, say. There is no choice of $c_{m 1}$ that will force $g(t) \equiv 0$. Therefore, $h_{m 0}(t)$ is not a polynomial.

We shall now examine the complete solutions of (11). For $m=0$ there is no nonhomogeneous part, and, from the above argument, $g_{00}(t)=c_{00}$. For $m=1$, it is clear that $p_{10}(t)=c_{00}$ and $g_{10}(t)=c_{10} t+c_{00}$. For simplicity we shall choose $c_{10}=0$. For $m=2$ we obtain $g_{20}(t)=c_{20} t^{2}+c_{00}$. We shall set $c_{20}=0$. Continuing in this manner we have $g_{m 0}(t)=c_{00}, m=1, \ldots, 6$. Then $g_{70}(t)=c_{70} t^{7}+c_{71} t^{\alpha_{7}+i \gamma_{7}}+c_{72} t^{\alpha_{7}-i \gamma_{7}}+c_{00}$. For simplicity, choose $c_{70}=c_{72}=0$.

For $m=8$, the right-hand side of (11) becomes

$$
\begin{aligned}
-\mu_{8}^{-1} & {\left[c_{00} \sum_{j=0}^{7} h_{8 j}+h_{87}\left[120 c_{71} t^{\alpha_{7}+i \gamma_{7}}+600 c_{71}\left(\alpha_{7}+i \gamma_{7}\right) t^{\alpha_{7}+i \gamma_{7}}\right.\right.} \\
& +600 c_{71}\left(\alpha_{7}+i \gamma_{7}\right)\left(\alpha_{7}+i \gamma_{7}-1\right) t^{\alpha_{7}+i \gamma_{7}} \\
& +200 c_{71} \prod_{j=0}^{2}\left(\alpha_{7}-j+\gamma_{7}\right) t^{\alpha_{7}+i \gamma_{7}} \\
& \left.\left.+25 c_{71} \prod_{j=0}^{3}\left(\alpha_{7}-j+\gamma_{7}\right) t^{\alpha_{7}+i \gamma_{7}}+c_{71} \prod_{j=0}^{4}\left(\alpha_{7}-j+\gamma_{7}\right) t^{\alpha_{7}+i \gamma_{7}}\right]\right] \\
= & -\mu_{8}^{-1}\left[c_{00}\left(1-\mu_{8}\right)+\lambda_{7} t^{\alpha_{7}+i \gamma_{7}}\right], \text { say. }
\end{aligned}
$$

Since $t^{\alpha_{7}+i \gamma_{7}}$ is a solution of the homogeneous part of (11) for $m=7$, it follows that $p_{80}(t)=c_{00}+a_{8} t^{\alpha_{7}+i \gamma_{7}}$, where $a_{8}=-\lambda_{7} \mu_{8}^{-1} /\left(\mu_{7}^{-1}-\mu_{8}^{-1}\right)=$ $\lambda_{7} \mu_{7} /\left(\mu_{7}-\mu_{8}\right)$. Thus $g_{80}(t)=c_{82} t^{\alpha_{8}+i \gamma_{8}}+c_{00}+a_{8} t^{\alpha_{7}+i \gamma_{7}}$, where, for simplicity we have set $c_{80}=c_{82}=0$. Continuing in this manner,

$$
g_{m 0}(t)=c_{m 1} t^{\alpha_{m}+i \gamma_{m}}+c_{00}+\sum_{j=7}^{m-1} a_{j} t^{\alpha_{j}+i \gamma_{j}} .
$$

Now choose $c_{m 1}=1 / m^{2}\left|\prod_{j=0}^{4}\left(\alpha_{m}-j+\gamma_{m}\right)\right|$. Equating like coefficients in (13) and (3), for $m>7$,

$$
a_{m 0}=c_{m 1}+c_{00},
$$

and, for $k>0$,

$$
a_{m k}=c_{m 1} \frac{\Gamma\left(k-\alpha_{m}-i \gamma_{m}\right)}{k !}+\sum_{j=7}^{m-1} a_{7} \frac{\Gamma\left(k-\alpha_{j}-i \gamma_{j}\right)}{k !},
$$

$\left|a_{m k}\right| \sim k^{-17 / 2}$, and $\|A\|<\infty$. (From (12) the sum of the roots is equal to $m+15$; i.e., $\alpha_{m}+\beta_{m}=(m+15) / 2$. Since $\beta_{m}<0$, each $\alpha_{m}>15 / 2$.) Clearly, $\lim _{m} a_{m 0}=0$. Since $c_{m 1}=O(1) / m^{2}$ and $g_{j}=O(1) / j^{2}, \lim _{m} a_{m k}=0$ for each $k>0$.

It is of interest to note that, if $A, H \in \mathscr{B}, H$ has distinct diagonal entries, and $A$ commutes with $H$, then the row sums of $A$ are equal to the same 
constant. To see this, we shall prove that $g_{n 0}(0)=g_{00}(0)$ for all integers $n>0$. From (3), $g_{n 0}(0)=\sum_{j=0}^{\infty} a_{n k}$. It is trivially true that $g_{00}(0)=g_{00}(0)$. Assume the induction hypothesis. Then, from (4),

$$
\int_{0}^{1} g_{n 0}(0) d q(u)=\sum_{j=0}^{n-1} h_{n j} g_{j 0}(0)
$$

i.e., $g_{n 0}(0) \mu_{0}=\mu_{n} g_{00}(0)+g_{00}(0)\left(\mu_{0}-\mu_{n}\right)$. Since the diagonal entries are distinct, $g_{n 0}(0)=g_{00}(0)$.

Therefore, $A \in \Gamma$.

Theorem 2. Let $n$ be a positive integer. Then $\operatorname{Com}(H, n)$ in $\Gamma=\mathscr{H}$ iff $1 \leq n \leq 4$.

Proof. We shall assume that $n>1$, since $(H, 1)=(C, 1)$. (5) takes the form

$$
\frac{1}{\Gamma(n)} \int_{0}^{1} g_{m 0}(t u)(\log 1 / u)^{n-1} d u=\sum_{j=0}^{m} h_{m j} g_{j 0}(t)
$$

or

$$
\frac{1}{\Gamma(n)} \int_{0}^{t} g_{m 0}(s)(\log t / s)^{n-1} d s=\sum_{j=0}^{m} h_{m j} g_{j 0}(t) .
$$

It can be verified that the $k$ th derivative of the above equation is

$$
\frac{(n-1)(n-2) \cdots(n-k)}{\Gamma(n)} \int_{0}^{t} g_{m 0}(s)(\log t / s)^{n-k-1} d s=\sum_{j=0}^{m} h_{m j} t(D t)^{k}\left(g_{j 0}(t)\right),
$$

where $D:=d / d t$.

With $k=n-1$ one obtains

$$
\int_{0}^{t} g_{m 0}(s) d s=\sum_{j=0}^{m} h_{m j} t(D t)^{n-1}\left(g_{j 0}(t)\right) .
$$

One more differentiation yields

$$
g_{m 0}(t)=\sum_{j=0}^{m} h_{m j}(D t)^{n}\left(g_{j 0}(t)\right)=\mu_{m}(D t)^{n}\left(g_{m 0}(t)\right)+\sum_{j=0}^{m-1} h_{m j}(D t)^{n}\left(g_{j 0}(t)\right) .
$$

It can be shown by induction that

$$
(D t)^{n}\left(g_{m 0}(t)\right)=\sum_{j=0}^{n} b(n, j) t^{j}\left(g_{m 0}^{(j)}(t)\right) .
$$

Therefore, (14) is an Euler type differential equation. Substituting $g_{m 0}(t)=r^{t}$ yields

$$
(r+1)^{n} t^{r}=(m+1)^{n} t^{r}-(m-1)^{n} \sum_{j=0}^{m-1} h_{m j}(D t)^{n}\left(g_{j 0}(t)\right) .
$$

The homogeneous part of equation (15) takes the form $(1+r)^{n}=(m+1)^{n}$. Thus, $r=-1+(m+1) e^{2 \pi i j / n}, j=0,1, \ldots, n-1$. 
For $n=2, r=m,-m-2$. If $h_{m 0}(t)$ denotes the homogeneous solution of (15), then $h_{m 0}(t)=c_{m 0} t^{m}+c_{m 1} t^{-m-2}$. Since $h_{m 0}(t)$ is continuous at $t=0$, we must have $c_{m 1}=0$. It then follows that $g_{m 0}(t)$ is a polynomial and $A$ is triangular.

For $n=3, r=m,-(m+3) / 2 \pm \gamma i$, where $\gamma=(m+1) \sqrt{3} / 2$, and $h_{m 0}(t)=$ $c_{m 0} t^{m}+c_{m 1} t^{-(m+3) / 2+\gamma i}+c_{m 2} t^{-(m+3) / 2-\gamma i}$. Using the argument of the case $n=3$ for Theorem 1, it follows that $c_{m 1}=c_{m 2}=0$, and $h_{m 0}(t)$ is a polynomial of degree $m$. Then $g_{m 0}(t)$ is a polynomial of degree $m$ and $A$ is triangular.

For $n=4, r=m,-1+(m+1) e^{2 \pi i j / 4}, j=0,1,2,3$, and

$$
h_{m 0}(t)=c_{m 0} t^{m}+c_{m 1} t^{-m-2}+c_{m 2} t^{-1+(m+1) i}+c_{m 3} t^{-1-(m+1) i} .
$$

Using an argument similar to that for the case $n=4$ of Theorem 1 , one can show that $c_{m 1}=0$, and then that $c_{m 2}=c_{m 3}=0$. It then follows that $g_{m 0}(t)$ is a polynomial of degree $m$ and $A$ is triangular.

For $n>4, r$ has roots in the first quadrant. Arguing as before it can be shown that those roots lying in the second and third quadrants must have coefficients zero. For convenience one can set $c_{m 0}=0$ and the coefficients of the roots in the fourth quadrant equal to zero. Using an argument similar to that for case $n=5$ of Theorem 1, one can show that $A$ is nontriangular, $A \in \Gamma$, and the commutant of $(H, n)$ in $\Gamma$ contains nontriangular matrices.

Theorem 3. $\operatorname{Com}(E, q)$ in $\mathscr{B}=\mathscr{H}$.

Proof. The Euler matrix $(E, q)$ is a regular Hausdorff matrix with

$$
q(u):= \begin{cases}0, & 0 \leq u<a, \\ 1, & a \leq u \leq 1,\end{cases}
$$

where $q$ and $a$ are related by the equation $q=(1-a) / a$.

From (5) we have (since $\mu_{m}=a^{m}$ )

$$
P_{m-1}(t)+a^{m} g_{m 0}(t)=P_{m-1}(t)+\mu_{m} m g_{m 0}(t)=\int_{0}^{1} g_{m 0}(t u) d q(u)=g_{m 0}(a t) .
$$

Differentiating the above equation $m$ times yields $g_{m 0}^{(m)}(t)=g_{m 0}^{(m)}(a t)$. Replacing $t$ successively by $a t$ in this equation leads to

$$
g_{m 0}^{(m)}(t)=g_{m 0}^{(m)}(a t)=\cdots=g_{m 0}^{(m)}\left(a^{n} t\right) .
$$

Taking the limit as $n \rightarrow \infty$ gives $g_{m 0}^{(m)}(t)=g_{m 0}^{(m)}(0)=\sum_{k=0}^{\infty} a_{m k}$, which implies that $g_{m 0}^{(m)}(t)$ is a constant over $[0,1]$. Therefore, $g_{m 0}(t)$ is a polynomial of degree $m$, and $A$ is triangular.

Theorem 4. If $q(u)=u^{p}, \operatorname{Re}(p)>0$, then $\operatorname{Com} H$ in $\Gamma=\mathscr{H}$.

Proof. In this case (5) becomes

$$
P_{m-1}(t)+\mu_{m} g_{m 0}(t)=\int_{0}^{1} g_{m 0}(t u) p u^{p-1} d u=\int_{0}^{t} g_{m 0}(s) p(s / t)^{p-1} d s / t,
$$

or

$$
\left(P_{m-1}(t)+\mu_{m} g_{m 0}(t)\right) t^{p}=p \int_{0}^{t} g_{m 0}(s) s^{p-1} d s
$$


Differentiating, one obtains

$$
p\left(P_{m-1}(t)+\mu_{m} g_{m 0}(t)\right) t^{p-1}+\left(P_{m-1}^{\prime}(t)+\mu_{m} g_{m 0}^{\prime}(t)\right) t^{p}=p g_{m 0}(t) t^{p-1},
$$

or

$$
\mu_{m} t g_{m 0}^{\prime}(t)+p\left(\mu_{m}-1\right) g_{m 0}(t)=-p P_{m-1}(t)-t P_{m-1}^{\prime}(t) .
$$

The particular nonhomogeneous solution of (16) is a polynomial of degree at most $m-1$. The homogeneous part of $(16)$ is an Euler-type differential equation with indicial equation $\mu_{m}(p+r)=p$ and $r=m$. Therefore, $A$ is triangular.

Thus far the only Hausdorff matrices to be considered have satisfied the condition that the diagonal entries form a totally monotone sequence. It is a reasonable question to ask if total monotonicity is a necessary condition. The answer is in the negative, and will be demonstrated by examining the commutant in $\Gamma$ of the known Hausdorff primes.

The convergence domain of an infinite matrix $A$ is the set of all sequences which $A$ maps into convergent sequences. A matrix $A$ is said to be stronger than a matrix $B$ if $A$ has the larger convergence domain. A multiplicative Hausdorff matrix $H_{1}$ is called a Hausdorff prime if (1) the convergence domain of $H_{1}$ properly contains $c$, but (2) if $H_{2}$ is a Hausdorff matrix such that $H_{1}$ is strictly stronger than $\mathrm{H}_{2}$, then the convergence domain of $\mathrm{H}_{2}$ is $\mathrm{c}$.

Using this definition of prime, it follows from [9] that all Hausdorff matrices generated by $\{(n-a) /(n+b)\}$ for $\operatorname{Re}(a), \operatorname{Re}(b)>0$ are primes. A longstanding open question is whether all primes are of this form.

Theorem 5. Let $H$ be a known Hausdorff prime. Then $\operatorname{Com} H$ in $\Gamma=\mathscr{H}$.

Proof. The mass function for $H$ is

$$
q(u)= \begin{cases}-(a+b) u^{b} / b, & 0 \leq u<1, \\ 1-(a+b) / b, & u=1 .\end{cases}
$$

Then

$$
\mu_{n}=1-\frac{(a+b)}{n+b} .
$$

Let $c, d \in \mathbb{C}, c d \neq 0, A, B$ infinite matrices, $I$ the unit matrix. Then $A B=$ $B A$ if and only if $A(c I+a B)=(c I+d B) A$. From Theorem 4, the commutant of $H_{\lambda}$ in $\Gamma, \lambda=b /(n+b)$, consists only of triangular matrices. Therefore, the commutant of $H_{\mu}$ in $\Gamma$ contains only triangular matrices.

Let $H$ denote the Hausdorff matrix generated $\mu_{m}=a /(n+a)$. Then it can be shown that the commutant of both $H(C, 1)^{-1}$ and $(C, 1) H^{-1}$ is $\mathscr{H}$. Also the commutant of $(H, 2)(C, 2)^{-1}$ and $(C, 2)(H, 2)^{-1}$ is $\mathscr{H}$. But the commutants of both $(H, 3)(C, 3)^{-1}$ and $(C, 3)(H, 3)^{-1}$ contain nontriangular matrices.

Let $B(c)$ denote the algebra of all bounded linear operators on $c$. Then an operator $T \in B(c)$ is of the form $T=v \otimes \lim +B$, where $B$ is the matrix representation of the restriction of $T$ to $c$, the space of null sequences, and $v$ is the bounded sequence $\left\{X_{i}(T)\right\}$, where $X_{i}(T):=(T e)_{i}-\sum_{k}\left(T e^{x}\right)_{i}, e=$ $\{1,1, \ldots\}$. Also $v \otimes \lim x=(\lim x) v$ for each $x \in c$. (See, e.g., [8].)

Since $B(c)$ strictly contains $\Gamma$, it is of interest to determine the commutant of a Hausdorff matrix in $B(c)$. In [7] it was shown that, if $H$ is a multiplicative 
Hausdorff matrix, $T \in B(c)$, then $T$ commutes with $H$ if and only if $T=$ $\lambda e \otimes \lim +B$ for some scalar $\lambda$, where $B$ commutes with $H$.

Thus, for any Hausdorff matrix discussed in this paper, the commutant in $B(c)$ is known.

\section{ACKNOWLEDGMENTS}

1. In 1987, during the author's visit to the University of Birmingham, he was informed by Dr. Kuttner that he had discovered that the commutant of $(C, \alpha)$, for $\alpha>4$, contains nontriangular matrices. Kuttner did not provide the author with a proof of this fact, nor did he give any indication how he had arrived at the result. Kuttner made no statements concerning the commutants of the other Hausdorff matrices considered in this paper. The author has recently received a preprint of a paper by Kuttner and Parameswaran (written after Kuttner's death, January 2, 1992) in which they show that the commutant in $\mathscr{M}$ of $(C, \alpha)$ is the set of Hausdorff matrices for $0<\alpha<4$, and, for $\alpha>4$, the commutant contains nontriangular matrices. The method of proof is different from that used in this paper, and treats only Cesàro matrices. Their positive results are stronger than Theorem 1, but the negative results are weaker. The author acknowledges a debt to Dr. Kuttner for having mentioned the commutant problem for $(C, \alpha)$.

2. The author takes this opportunity to thank his colleague Grahame Bennett for several valuable conversations.

3. The author wishes to thank the referee for his careful reading of the manuscript and for detecting an error in the original proof of Theorem 1.

Open questions. 1. Are there any other conservative Hausdorff matrices, with distinct diagonal entries, not covered by the theorems of this paper, whose commutants in $\mathscr{B}$ are $\mathscr{H}$ ?

2. Is $\operatorname{Com}(H, \alpha)$ in $\mathscr{B}=\mathscr{H}$ for $0<\alpha<4$ ?

3. Does $\operatorname{Com}(C, \alpha)$ in $\mathscr{B}$ contain nontriangular matrices for all $\alpha>4$ ?

4. Does $\operatorname{Com}(H, \alpha)$ in $\mathscr{B}$ contain nontriangular matrices for all $\alpha>4$ ?

\section{REFERENCES}

1. Stephen Barnett, Polynomials and linear control systems, Marcel Dekker, New York and Basel, 1983.

2. F. Hausdorff, Summationmethoden und momentfolgen, Math. Z. 9 (1921), 74-109.

3. J. D. Hill, On perfect methods of summability, Duke Math. J. 3 (1937), 702-714.

4. A. Jakimovski, The product of summability methods: New classes of transformations and their properties, Tech. Note Contract AF 61 (052)-187, U.S. Air Force.

5. B. Kuttner and M. R. Parameswaran, Matrices that commute with $(C, \alpha)$, preprint.

6. B. E. Rhoades, Commutants of some Hausdorff matrices, Pacific J. Math. 42 (1972), 715-719.

7. $\longrightarrow$ Correction to: Commutants of some Hausdorff matrices, Pacific J. Math. 49 (1973), 617-619.

8. B. E. Rhoades and A. Wilansky, Some commutants in $B(c)$ which are almost matrices, Pacific J. Math. 49 (1973), 211-217.

9. L. L. Silverman and J. D. Tamarkin, On the generalization of Abel's theorem for certain definitions of summability, Math. Z. 29 (1928), 161-170. 